\title{
THE KLUVANEK-KANTOROVITZ CHARACTERIZATION OF SCALAR OPERATORS IN LOCALLY CONVEX SPACES
}

\author{
WILLIAM V. SMITH \\ Mathematics Department \\ The University of Mississippi \\ University, Mississippi 38677 \\ (Received June 9, 1980)
}

ABSTRACT. This paper is devoted to a proof of the characterization without duality theory, using strong integrals, while eliminating any assumptions of barrelledness or equicontinuity.

KEY WORDS AND PHRASES. Locally convex spaces, scalar operators, Banach space. 1980 MATHEMATICS SUBJECT CLASSIFICATION CODES. $47 B 40 ; 28 B 05$.

1. INTRODUCTION.

This paper is directed to specialists familiar with its field. In [1] we will indicate that some results regarding the characterization of scalar type operators in Banach spaces can be generalized to the locally convex case rather simply. In this note we will indicate briefly how this can be done with a result of Kluvanek [2] (see Kantorovitz [3]).

Kluvanek's paper [2] is concerned with a problem somewhat more general than the one we will look at but the interested reader will see that we could carry out his program by the same technique as used here.

Perhaps the most useful aspect of our work here is the fact that we are able to construct the proofs almost entirely without duality theory. The use of linear functionals is kept to a minimum, and while some of the results (especially some lemmas) appear to depend on local convexity, this can even be avoided as in [1]. However, local convexity is apparently necessary to avoid certain problems with some of the integrals involved and to allow the use of results in [4]. The reader 
will also note that the spaces involved are not assumed to be barrelled nor the spectral measures equicontinuous. This is an improvement over previous work in the area.

\section{NOTATION AND PRELIMINARIES.}

$\mathrm{L}(\mathrm{X})$ will be the continuous endomorphisms of a quasicomplete separated locally convex topological vector space $X$; we assume $L(X)$ is quasicomplete for simple convergence. $S$ will be a locally compact Hausdorff space and $C_{0}(S)$ will be the continuous complex valued functions on $S$ which vanish at infinity. $\|f\|_{\infty}$ will denote the supremum norm of $f$, and $\hat{f}$ will denote the Fourier transform of a function $f$ on $\mathbf{R}^{\prime}$ the real line.

LEMMA 2.1. Let $B$ be the Borel subsets of $S$. Suppose $P: B \rightarrow L(X)$ is a regular countably additive set function and $D$ is a dense subalgebra of $C_{0}(S)$. If $S$ is a metric space and

$$
\int f g d P=\left(\int f d P\right)\left(\int g d P\right)
$$

for $f, g \in D$, then $P$ is multiplicative (i.e., $P\left(E_{1} \cup E_{2}\right)=P\left(E_{1}\right) P\left(E_{2}\right)=P\left(E_{2}\right) P\left(E_{1}\right)$ ). PROOF OF LEMMA 2.1. The proof may be done using the fact that $P$ has bounded semivariation and by considering the argument in (2.5) of [1].

LEMMA 2.2. Let $D$ be as in Lemma 2.1. and consider $\Phi: D \rightarrow X$. Suppose

$$
(*)=\left\{\Phi(f) \mid\|f\|_{\infty} \leq 1, f \in D\right\}
$$

is relatively weakly compact in $\mathrm{X}$.

Then $\Phi$ is a continuous operator and there is a unique X-valued countably additive regular set function $m$ such that

$$
\Phi(f)=\int f d m
$$

for $f \in D$. Conversely, if such an $m$ exists, then $(*)$ is relatively weakly compact. PROOF OF LEMMA 2.2. See Lewis [5], page 164; or, when [6] appears, use Theorem 4 with the proof of Lemma 2 in [2].

REMARK. The fact that $\Phi$ is continuous on all of $\mathrm{C}_{0}(S)$ can be inferred from the fact that in a locally convex space weakly bounded sets are bounded and from the fact that bounded linear maps from an F-space to a topological vector space are continuous . 


\section{CHARACTERIZATION.}

THEOREM 3.1. Let $T \in L(X)$. $T$ is scalar with real spectrum if and only if

$$
(+)=\left\{\int_{-\infty}^{\infty} f(s) e^{i s T} x d s \mid\|\hat{f}\|_{\infty} \leq 1, f \in L^{l}(-\infty, \infty)\right\}
$$

is a relatively weakly compact subset of $\mathrm{X}$ for $\mathrm{x} \varepsilon \mathrm{X}$.

PROOF OF THEOREM 3.1. The map $\hat{f} \rightarrow \int f(s) e^{i s T}$ ds is continuous and weakly compact and, therefore, by Lemma 2.2, $\int_{-\infty}^{\infty} f(s) e^{i s T} x d s=\int \hat{f} d \mu_{x}$ for each $x \varepsilon x$. If $\mathbf{x}^{\prime} \varepsilon \mathrm{X}^{\prime}$ the continuous dual of $\mathrm{X}$ then recalling that $\mu_{\mathbf{X}}$ is bounded

$$
\begin{aligned}
\int_{-\infty}^{\infty} f(s)\left(e^{i s T} x, x^{\prime}\right) d s & =\int \hat{f} d\left(\mu_{x}, x^{\prime}\right) \\
& =\iint_{-\infty}^{\infty} f(t) e^{-i s t} d t d\left(x^{(s)}, x^{\prime}\right) \\
& =\int_{-\infty}^{\infty} f(t) \int e^{-i s t} d\left(\mu_{x}(s), x^{\prime}\right) d t \\
& =\int_{-\infty}^{\infty} f(t) \int e^{-i s t} d\left(\mu_{x}(s), x^{\prime}\right) d t
\end{aligned}
$$

and, therefore,

$$
\left(e^{i t T} x, x^{\prime}\right)=\int e^{-i s t} d\left(\mu_{x}(s), x^{\prime}\right)
$$

by continuity of $e^{i t T} x$ in $t$. Since $e^{i s t}$ is bounded,

$$
e^{i t T} x=\int e^{-i s t} d \mu_{x}(s)
$$

Define $P(E)(x)=\mu_{x}(E)$. Then $P$ is $L(X)$ valued countably additive and regular (in the strong operator topology). Now it is easy to see that if $f * g(x)=$ $\int_{-\infty}^{\infty} f(x-y) g(y) d y$, then

$$
\int f * g(s) e^{i s t} x d s=\int f(t) e^{i t T} d t \int g(u) e^{i u T} x d u
$$

for all $f, g \in L^{l}\left(R^{1}\right)$. Therefore since $\widehat{f * g}=\hat{f} \hat{g},\left(P_{x}(E)=P(E) x\right)$

$$
\int \hat{f} \hat{g} d P_{x}=\int \hat{f} d P \int \hat{g}_{d P}
$$

for all $f, g \in L^{1}\left(R^{1}\right)$. Since $\widehat{L^{1}\left(\mathbf{R}^{1}\right)}$ is dense in $C_{0}\left(\mathbb{R}^{1}\right)$, by Lemma $2.2, P$ is multiplicative and setting $t=0$ in (3.1) gives $P\left(\mathbb{R}^{1}\right)=I$ the identity in $L(X)$. Hence $P$ is a spectral measure.

$$
\text { Notice } \frac{e^{i s t}-1}{i t} \rightarrow s \text { as } t \rightarrow 0 \text { and using Proposition (4.1) and Proposition (5.4) }
$$

of [7] we have (the proof of (5.4) is what is required)

$$
\mathrm{Tx}=-\int \lambda \mathrm{dP}_{\mathrm{x}}
$$


for all $x$ in a dense subset of $x$ and if $\delta_{n}=[-n, n]$, then it is clear that $\operatorname{TP}\left(\delta_{n}\right) x=$ $\mathrm{P}\left(\delta_{\mathrm{n}}\right) \mathrm{Tx} \rightarrow-\mathrm{Tx}$ for all $\mathrm{x}$ by continuity of $\mathrm{T}$ and since $\int \lambda \mathrm{dP}_{\mathrm{x}}$ is closed and $\lim _{n \rightarrow \infty} \int \lambda d P_{P}\left(\delta_{n}\right) x=-T x$ we have $-\int \lambda d P_{x}=T x$ for all $x$. Therefore, $T$ is scalar. Conversely, if $T x=-\int \lambda d P_{x}$, then $e^{i s T} x=\int e^{-i \lambda s} d P_{x}$ and one can show that $e^{i s T} x$ is continuous in $s$ and bounded as in [2]. Furthermore, $\int_{-\infty}^{\infty} f(s) e^{i s T} x d s$ exists as a Pettis integral by Thomas [4] for every $f \in L^{1}\left(\mathbf{R}^{l}\right)$, and this implies

$$
\hat{f} \rightarrow \int_{-\infty}^{\infty} f(s) e^{i s T} x d s=\iint_{-\infty}^{\infty} f(s) e^{-i s \lambda} d P(\lambda) x^{d s}
$$

or

$$
\begin{aligned}
\left(\int f(s) e^{i s T} x d s, x^{\prime}\right) & =\left(\iint f(s) e^{-i s \lambda} d P_{x}(\lambda) d s, x^{\prime}\right) \\
& =\iint f(s) e^{-i s \lambda} d\left(P_{x}(\lambda), x^{\prime}\right) d s \\
& =\iint f(s) e^{-i s \lambda} d s d\left(P(\lambda)_{x}, x^{\prime}\right)
\end{aligned}
$$

and so $\int f(s) e^{i s T} x d s=\int \hat{f}(\lambda) d P_{x}(\lambda)$ and, therefore, $(+)$ is relatively weakly compact by Lemma 2.2. This completes the proof.

A very well-known result of Bochner is:

THEOREM 3.2. If $\phi(t)$ is continuous for $-\infty<t<\infty$ and has the property that

$$
\left|\sum_{r=1}^{n} c_{r} \phi\left(t_{r}\right)\right| \leq K \sup _{z \varepsilon R}\left|\sum_{r=1}^{n} c_{r} e^{z i t} r\right|
$$

holds for all finite complex sequences $\left\{c_{r}\right\}$ and rational sequences $\left\{t_{r}\right\}$ then there exists a complex measure $v$ s.t.

$$
\phi(t)=\int_{-\infty}^{\infty} e^{i t z} d v(z),\|v\|<K .
$$

Using this result and the methods of 3.1. above and Lemma 7 of [3], we obtain the following (we omit the proof).

THEOREM 3.3. Suppose the operator $T \in L(X)$ satisfies the following where $\mathrm{X} \varepsilon \mathrm{A}$ a bounded subset of $\mathrm{X}$ and $\mathrm{X}^{\prime} \varepsilon \mathrm{A}^{\prime}$ an equicontinuous subset of $\mathrm{X}^{\prime}$

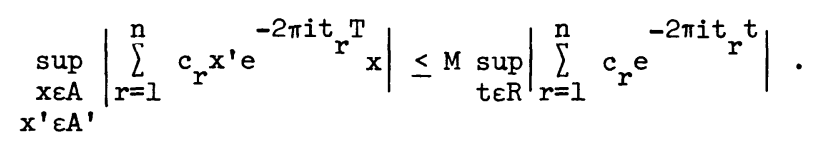

Suppose in addition that $\mathrm{X}$ is weakly sequentially complete. Then $\mathrm{T}$ is a scalar operator with real spectrum. 


\section{REFERENCES}

1. SMITH, W. V. Spectral Measures in Topological Algebras and Scalar Type Operators II (to appear), Per. Math. Hung.

2. KLUVANEK, I. Characterization of Fourier-Stieltjes Transforms of Vector and Operator Valued Measures, Czechoslovak Math. J. 17 (92), (1967), pp. 261277.

3. KANTOROVITZ, S. On the Characterization of Spectral Operators, Transactions of American Mathematical Society 111 (1964), pp. 152-181.

4. THOMAS, G. E. F. Totally Summable Functions with Values in Locally Convex Spaces, Measure Theory, Ober. (1975), Springer Lecture Notes, Berlin, (1976).

5. LEWIS, D. R. Integration with Respect to Vector Measures, Pacific J. Math. 33, pp. 157-165

6. SMITH, W. V. and D. H. TUCKER Weak Integral Convergence Theorems and Weak Compactness (to appear), Pacific J. Math.

7. SMITH, W. V. Spectral Measures in Topological Algebras and Scalar Type Operators I (to appear), Per. Math. Hung. 


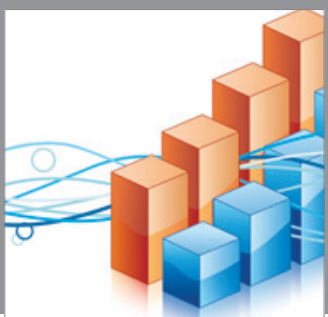

Advances in

Operations Research

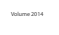

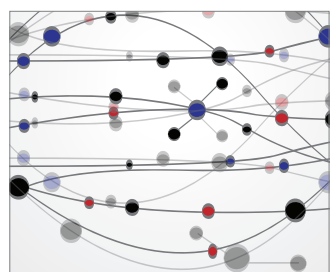

\section{The Scientific} World Journal
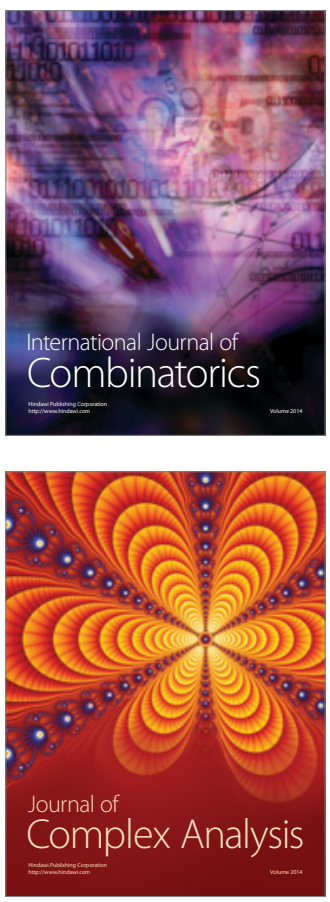

International Journal of

Mathematics and

Mathematical

Sciences
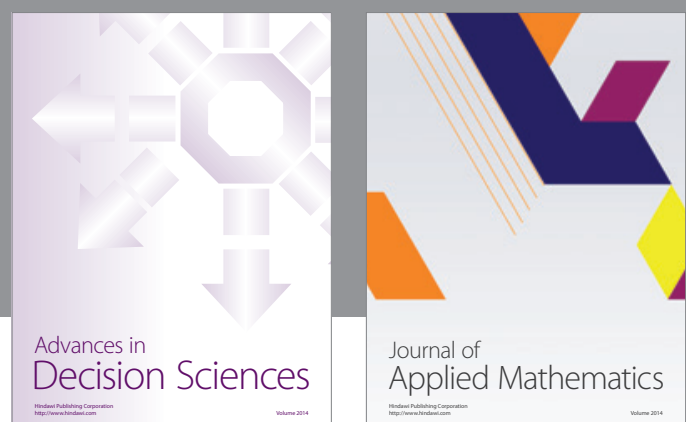

Journal of

Applied Mathematics
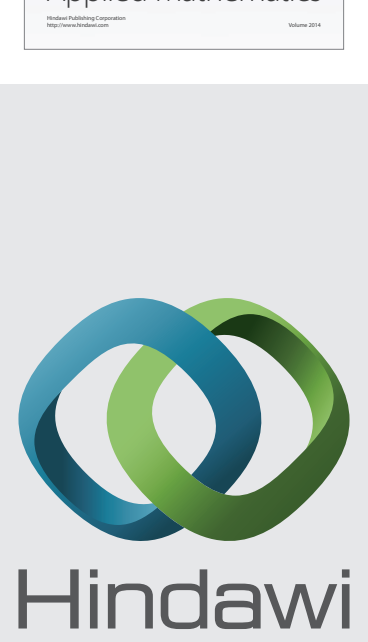

Submit your manuscripts at http://www.hindawi.com
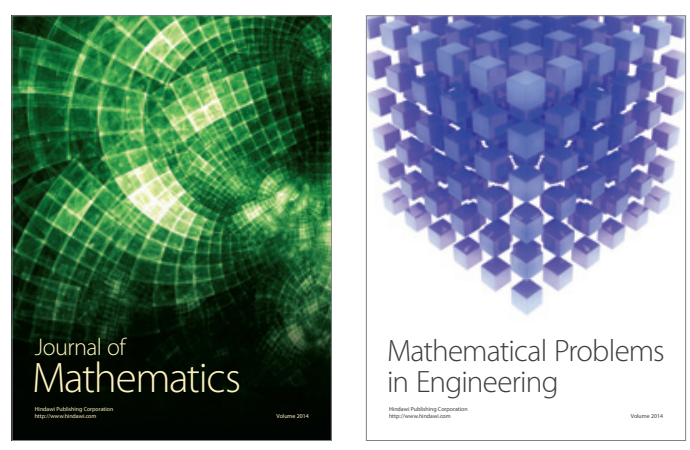

Mathematical Problems in Engineering
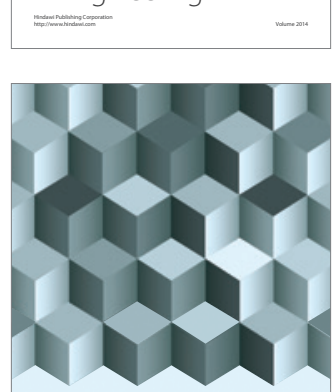

Journal of

Function Spaces
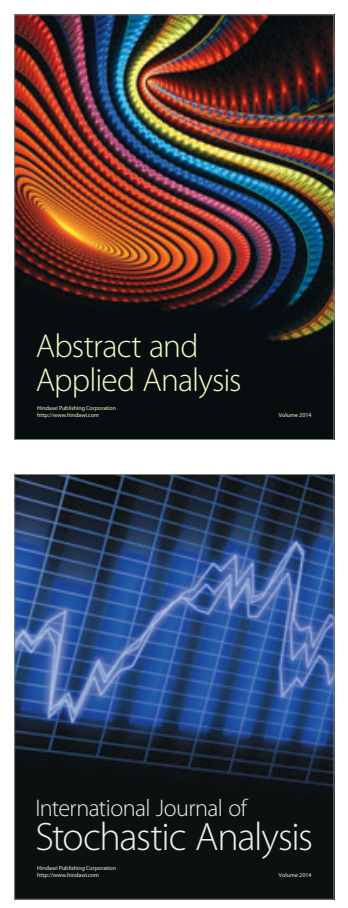

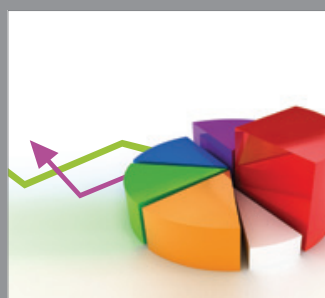

ournal of

Probability and Statistics

Promensencen
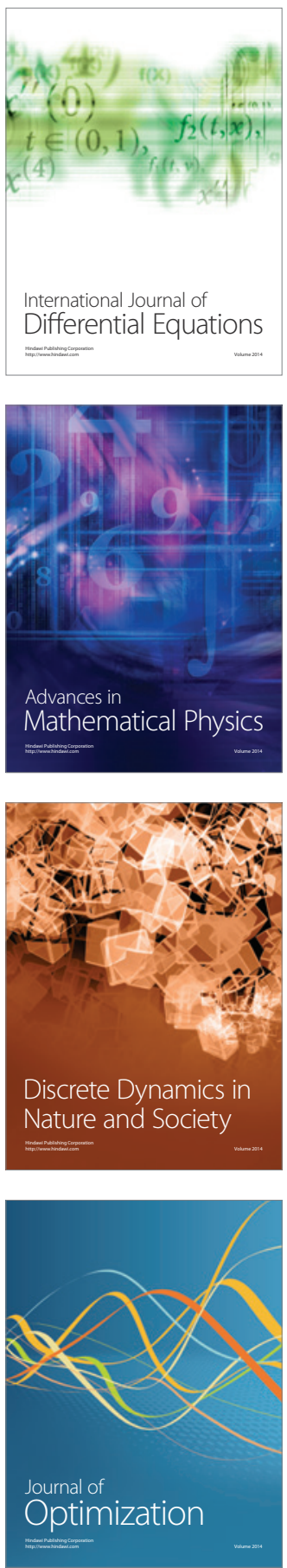